\title{
Origin of the Summertime Synoptic-Scale Wave Train in the Western North Pacific*
}

\author{
TIM LI \\ International Pacific Research Center and Department of Meteorology, University of Hawaii at Manoa, Honolulu, Hawaii
}

(Manuscript received 6 April 2005, in final form 7 September 2005)

\begin{abstract}
The origin of the summertime synoptic wave train in the western North Pacific is investigated with a multilevel, nonlinear baroclinic model. A realistic three-dimensional summer mean state is specified and eigenvectors are calculated by introducing small perturbation initially to the model. Numerical experiments indicate that the origin of the synoptic wave train may arise from instability of the summer mean flow in the presence of a convection-frictional convergence (CFC) feedback. In the lack of the CFC feedback, the summer mean flow supports only a least damped mode, characterized by a northwest-southeast-oriented wave train pattern with a zonal wavelength of $2500 \mathrm{~km}$. In the presence of both the realistic summer mean flow and the CFC feedback, the model reproduces a fast growing mode, whose structure and propagation characters are similar to the observed.

Sensitivity experiments with different initial perturbation patterns indicate that the model solution is not sensitive to initial conditions. Further sensitivity experiments reveal that the basic-state vertical shear may affect the growth rate and propagation character of the wave train. An easterly shear may lead to a faster growth and northwestward phase propagation, whereas a westerly shear may favor a slower growth and southeastward phase propagation.
\end{abstract}

\section{Introduction}

Two salient features of boreal summer tropical atmospheric circulation over the western North Pacific (WNP) are 1) a large-scale intertropical convergence zone (ITCZ)-monsoon trough pattern with active tropical cyclone (TC) activity and 2) the frequent reoccurrence of synoptic-scale wave trains oriented in the northwest-southeast direction (Lau and Lau 1990). Although previous studies have recognized a possible connection between the two phenomena (e.g., Chang et al. 1996), the physical mechanisms associated with the origin of the synoptic wave trains and their role in initiating tropical cyclogenesis have not been fully understood.

Lau and Lau (1990) first identified the northwestsoutheast oriented synoptic-scale wave trains based on the analysis of low-level vorticity and meridional wind

\footnotetext{
* School of Ocean and Earth Science and Technology Contribution Number 6715 and International Pacific Research Center Contribution Number 351.

Corresponding author address: Prof. Tim Li, IPRC, University of Hawaii at Manoa, 2525 Correa Rd., Honolulu, HI 96822. E-mail: timli@hawaii.edu
}

fields. The wave train extends toward the southeast with a wavelike pattern that consists of alternating regions of cyclonic and anticyclonic circulations propagating northwestward along the confluence zone of the mean summer flow and extending from the equatorial western Pacific to Southeast Asia, with some exceptions reaching the Indian subcontinent. The wave trains have a typical wavelength of $2500-3000 \mathrm{~km}$ and a time scale of 6-10 days.

Processes that trigger the synoptic-scale wave train include the barotropic instability of the tropical easterly flow (Nitta and Yanai 1969), Rossby wave energy dispersion of a preexisting mature TC (Holland 1995; Li et al. 2003; Li and Fu 2006; Li et al. 2006), an equatorialto-off-equatorial transition of mixed Rossby-gravity (MRG) waves (Takayabu and Nitta 1993; Liebmann and Hendon 1990; Dickinson and Molinari 2002), and scale contraction and energy accumulation of easterly waves (Tai and Ogura 1987; Kuo et al. 2001; Tam and Li 2006). Using a linear shallow-water model, Aiyyer and Molinari (2003, hereafter AM03) simulated the evolution of equatorial MRG waves in an idealized background state similar to the active phase of the Madden-Julian oscillation (MJO). Their numerical result suggests that the asymmetry in the background 
flow may favor the transfer of an equatorial perturbation to off-equatorial disturbances.

The numerical study by AM03 implies that the perturbation energy for the growth of the off-equatorial disturbances comes from the specified mean flow, as the model does not include a perturbation heating feedback. This seems different from the observational analysis by Lau and Lau (1992), who pointed out that the major energy source for the wave train growth comes from latent heating in the middle troposphere. In this study, we intend to examine the origin of the tropical synoptic wave train in a multilevel baroclinic model that specifies a realistic 3D summer mean flow. We are particularly interested in investigating whether the observed summer mean flow alone, without involving of the convection-circulation feedback, is able to lead to the growth of tropical disturbances. Our numerical experiments demonstrate that, while the mean flow (particularly its vertical shear) is essential to determine the wave train structure and propagation characters, the convection-circulation feedback is crucial to lead to the unstable growth.

The outline of the paper is as follows. In section 2 we briefly describe the model. In section 3 we present results from cases with and without the convective heating feedback. In sections 4 and 5 we examine the sensitivity of the model solution to initial conditions and to idealized vertical shear conditions. A conclusion and discussion are given in the final section.

\section{The model}

A modified version of the Princeton dynamic-core atmospheric general circulation model (AGCM) is employed for this study. The major difference between the current model and its original version (Held and Suarez 1994) lies in that the current model is linearized by a specified 3D basic state (e.g., the summer mean basic state) so that one may examine the evolution of a specified initial perturbation under an idealized or realistic mean basic state. The nonlinear baroclinic model is essentially the same as that of Ting and Yu (1998), and the perturbation equations retain full nonlinearity (see the appendix for the detailed derivation of the model and the treatment of circulation-dependent perturbation heating). Using this model, Wang et al. (2003) examined an equatorially asymmetric atmospheric response to a symmetric forcing, and Jiang and $\mathrm{Li}$ (2005) studied the reinitiation of MJO convection in the western Indian Ocean in boreal summer.

The global spectral model uses sigma, $\left(\sigma=p / p_{s}\right)$, as its vertical coordinate. There are five evenly distributed sigma levels with an interval of 0.2: a top level at $\sigma=0$ and a bottom level at $\sigma=1$. The horizontal resolution of T42 is adopted in this study. The basic equations in the model include momentum, temperature, and logarithm of surface pressure equations together with a diagnostic equation for the vertical velocity.

A biharmonic diffusion is applied to the momentum and temperature equations. Rayleigh friction is applied to the momentum equations, with the damping rate of 1 day $^{-1}$ taken in the lowest model level $(\sigma=0.9)$ to mimic the planetary boundary layer (PBL) linearly decaying to $0.1 \mathrm{day}^{-1}$ at the level of $\sigma=0.7$. Newtonian cooling with an $e$-folding time scale of 10 days is applied to the temperature equation at all model levels.

Since our focus is on tropical perturbations, a strong damping of 1 day $^{-1}$ is applied in the perturbation momentum and temperature equations over higher latitude regions (beyond $40^{\circ} \mathrm{N}$ and $40^{\circ} \mathrm{S}$ ). A realistic summer (June-August: JJA) mean state of $\bar{u}, \bar{v}, \bar{T}, \bar{P}_{s}$ is prescribed as the basic state, taken from the long-time mean of the National Centers for Environmental Prediction-National Center for Atmospheric Research (NCEP-NCAR) reanalysis (by linearly interpolating the original standard pressure level data to the model sigma levels).

\section{Roles of the summer mean flow and the convection-circulation feedback}

To test the hypothesis that the synoptic-scale wave train in the WNP results from the instability of the summer mean flow in the presence of the convectionfrictional convergence feedback, ${ }^{1}$ we take a numerical approach by examining how an initial perturbation evolves with time under a realistic 3D summer-mean basic state. For a control experiment, the initial perturbation has a zonal wavenumber-2 structure. Figure 1 shows the horizontal pattern of this initial perturbation. The initial vorticity perturbation has a weak amplitude (in an order of $10^{-8} \mathrm{~s}^{-1}$ ), and is confined in the lower troposphere (at $\sigma=0.7$ ).

Figure 2 shows the time evolution of the maximum perturbation kinetic energy (defined as a maximum value at each time step) and the amplitude of geopotential height fields at $\sigma=0.7$. In the presence of both the summer mean flow and the CFC feedback, the

\footnotetext{
${ }^{1}$ The CFC feedback represents a two-way interaction between free atmospheric circulation/convective heating and boundary layer flows as illustrated by Wang and Li (1993, 1994). On one hand, boundary layer moisture convergences impact the convective heating in the midtroposphere; on the other hand, the heating induces change of the free atmospheric circulation, which further impacts the boundary layer flows.
} 


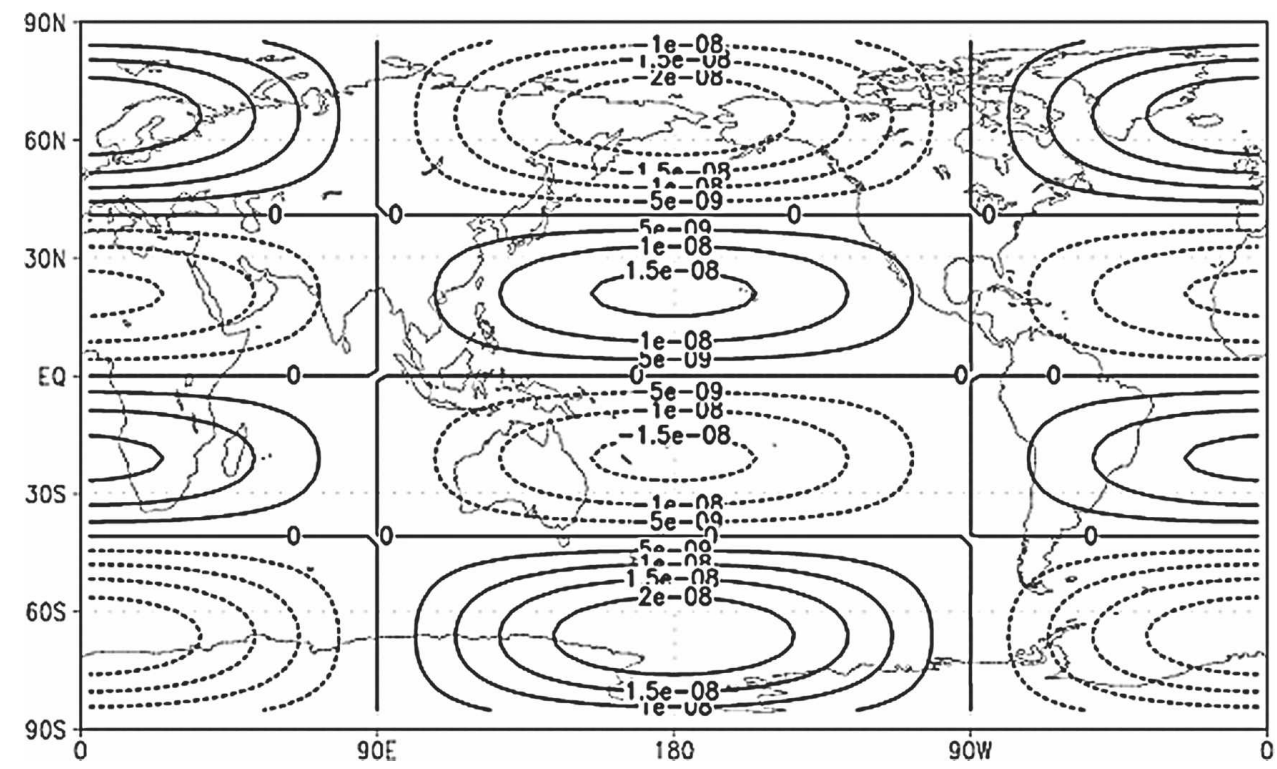

FIG. 1. Horizontal pattern of an initial vorticity perturbation (contour interval is $0.5 \times 10^{-8} \mathrm{~s}^{-1}$ ) in the control experiment.

model captures the growth of the most unstable mode. At day 5, the maximum perturbation wind speed reaches $2.5 \mathrm{~m} \mathrm{~s}^{-1}$. The growth rate of the unstable mode may be estimated based on the time series of the model wind, and the result shows that the growth rate is about $1.5 \mathrm{day}^{-1}$.

The fastest growing mode in the model has a typical observed synoptic-scale wave train structure in the WNP (see Fig. 3). The wave train, oriented in the northwest-southeast direction, has alternative cyclonic and anticyclonic circulation regions and a zonal wavelength of $2500 \mathrm{~km}$. The preferred geographic location for the perturbation growth appears in the WNP, primarily due to high mean SST and surface moisture content in the region. This is because, given the same perturbation vorticity, these background surface conditions would lead to the strongest CFC feedback.

The unstable mode, with an eastward-tilting vertical structure, propagates northwestward at a phase speed of about $10^{\circ}$ day $^{-1}$ (Fig. 4). Compared to the observed propagation speed of 5-8 $\mathrm{m} \mathrm{s}^{-1}$ (Lau and Lau 1990), the model phase speed is a little faster. Various factors may contribute to the northwestward phase propagation. First, the beta effect may lead to a westward tendency of perturbation vorticity. Second, under the CFC feedback scenario, a northward shift of cyclonic vorticity (and associated Ekman pumping and boundary layer convergence) relative to a convection center, caused by barotropic and baroclinic mode coupling under an easterly shear (Wang and Xie 1996; Jiang et al. 2004), may lead to a northward propagation tendency.
Third, the mean flow steering/advection may provide an additional northwestward propagation tendency.

Why does the most unstable mode prefer the synoptic-scale wavelength? To examine the possible role of the summer mean flow on the characteristic length scale selection, we conduct a sensitivity experiment by removing the $\mathrm{CFC}$ feedback (i.e., neglecting the perturbation heating by setting $\alpha=0$ ). The model is inte-

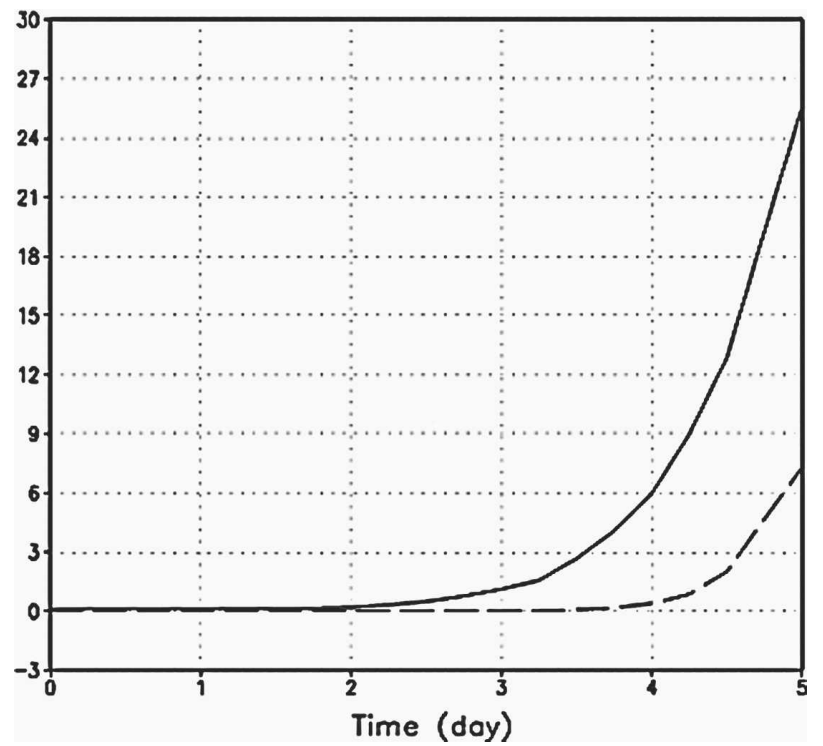

FIG. 2. Evolution of maximum perturbation kinetic energy (dashed line, unit is $\mathrm{m}^{2} \mathrm{~s}^{-2}$ ) and geopotential height (solid line, unit is $\mathrm{m}$ ) at $\sigma=0.7$ in the presence of both summer mean flow and CFC feedback. 
(a) Meridional wind

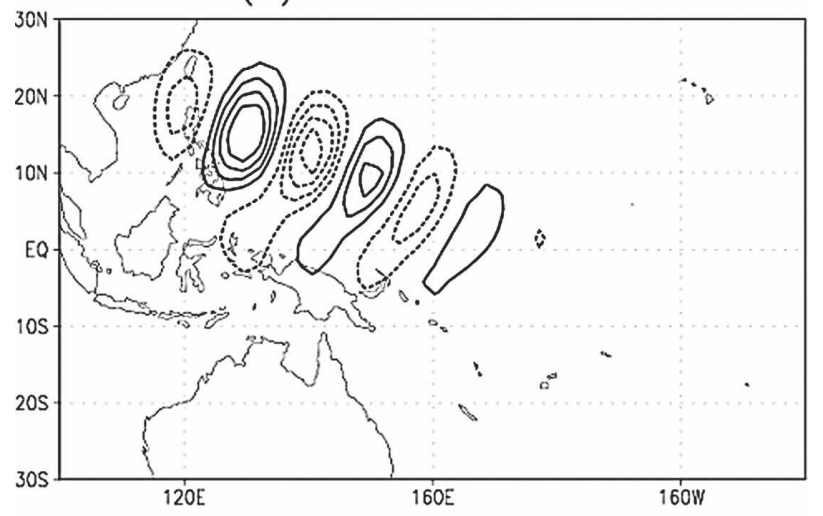

(b) Geopotential height

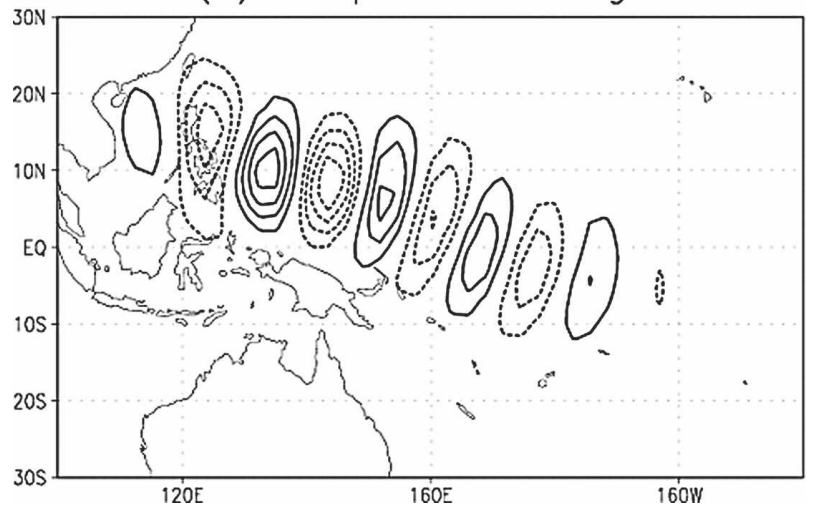

FIG. 3. Horizontal patterns of the most unstable mode at day 5: (a) normalized meridional wind field and (b) normalized geopotential height field at $\sigma=0.7$ (contour interval is 0.2 ).

grated for 25 days. The same initial perturbation as in the control experiment is used. Figure 5 shows the evolution of the maximum perturbation kinetic energy in this case. In the absence of the CFC feedback, the perturbation does not grow; rather it decays. This indicates that the climatological summer mean flow alone (without involving of the convective feedback) is unable to destabilize tropical perturbations. Thus, the summer mean flow only supports a weakly damped mode in the Tropics. This differs fundamentally from midlatitude baroclinic instability waves, which gain energy directly from the mean westerly jet. The numerical result is consistent with the previous observational study by Lau and Lau (1992), who pointed out that the primary heat source for the synoptic wave train is the latent heating associated with penetrated convection.

It is noted from Fig. 5 that the maximum kinetic energy experiences an oscillatory decay with two peaks appearing at day 4 and day 10 , respectively; so does the maximum perturbation geopotential height (figure not shown). This implies that the mean flow may play a role (a) Vertical structure of geopotential height

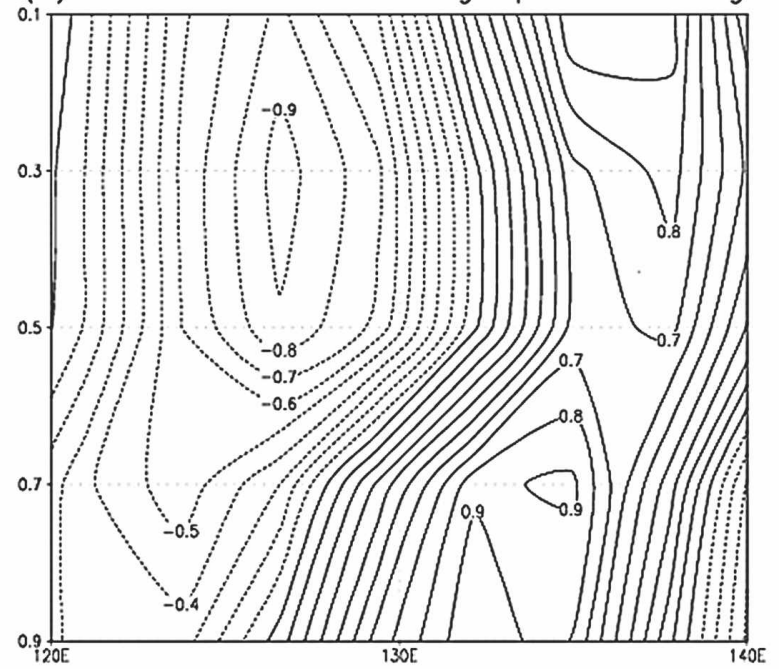

(b) Geopotential Height of $10 \mathrm{~N}$

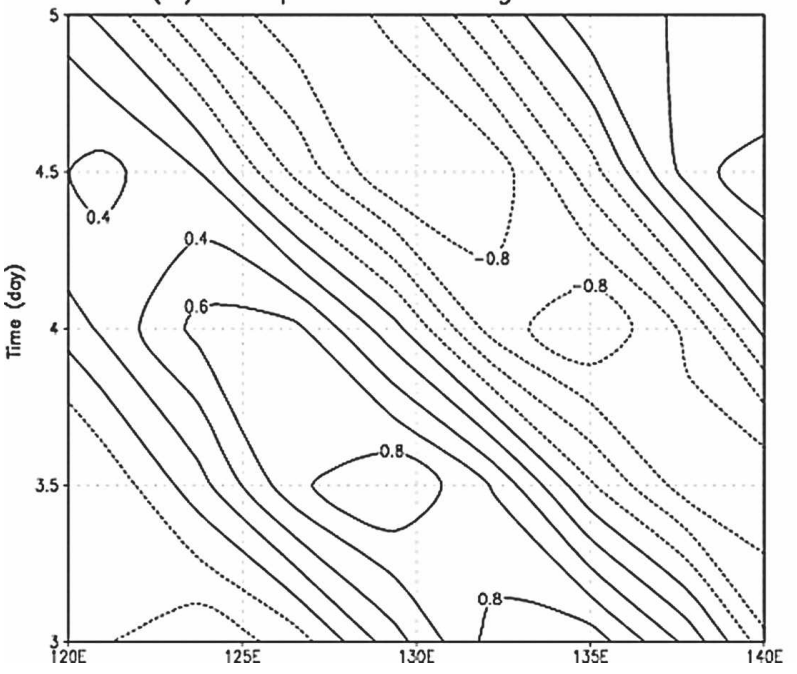

FIG. 4. (a) Vertical structure of the normalized geopotential height field at day 5 and (b) time-longitude section of the normalized geopotential height field from day 3 to day 5 along $10^{\circ} \mathrm{N}$.

in accumulating perturbation energy, possibly through wave flux convergences in a confluent flow zone (Kuo et al. 2001).

Even though the climatological summer mean flow alone is unable to lead to the perturbation growth, it does play a role in determining the preferred length scale, wave train structure, and propagation characteristics. Figure 6 illustrates the meridional wind structure of the least damped mode in the model. A northwestsoutheast oriented wave train pattern emerges. The wave train has alternative cyclonic and anticyclonic circulation regions with a wavelength of about $2500 \mathrm{~km}$.

The weakly damped mode propagates toward the 


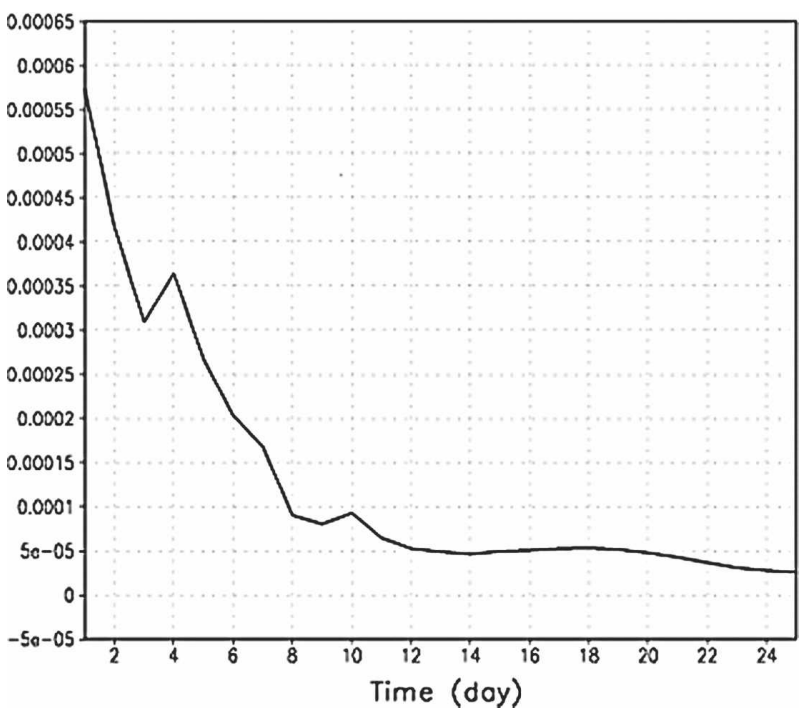

FIG. 5. Time evolution of maximum perturbation kinetic energy (unit is $\mathrm{m}^{2} \mathrm{~s}^{-2}$ ) at $\sigma=0.7$ in the lack of CFC feedback. A climatological summer mean flow is specified as the model basic state.

west and northwest. Figure 7 illustrates the longitudetime section of the meridional wind along $10^{\circ} \mathrm{N}$. The propagation speed is about $2.5^{\circ} \mathrm{day}^{-1}$, which is somewhat slower than the observed and that derived from the control experiment.

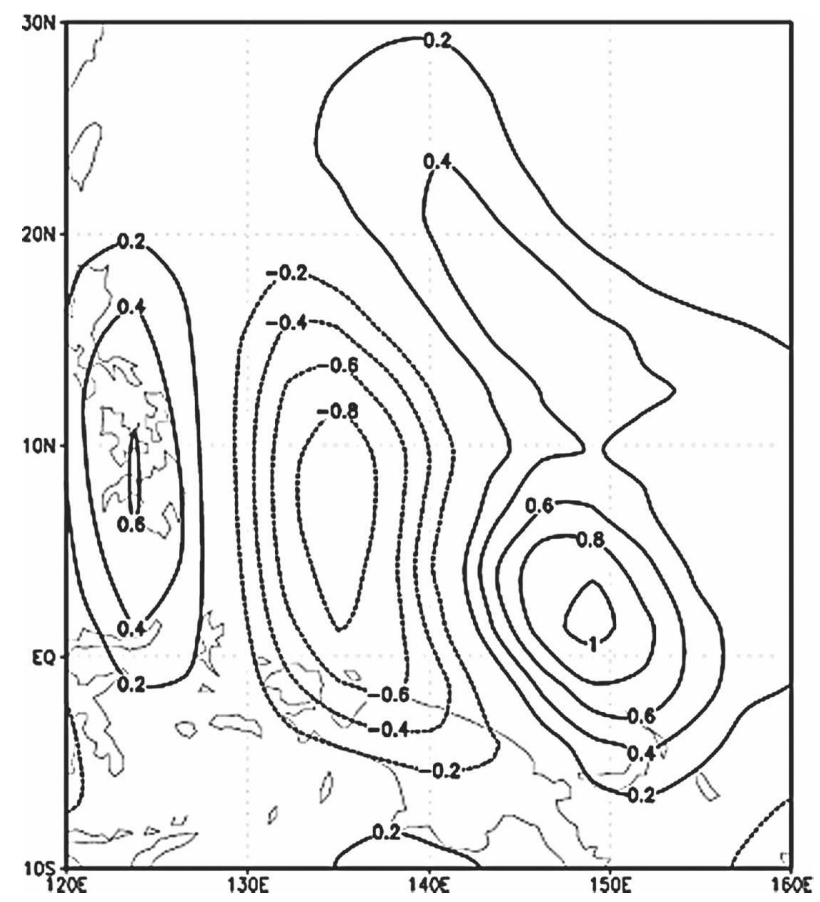

FIG. 6. Normalized (divided by its maximum amplitude) meridional wind pattern of the least damped mode at day 25 .

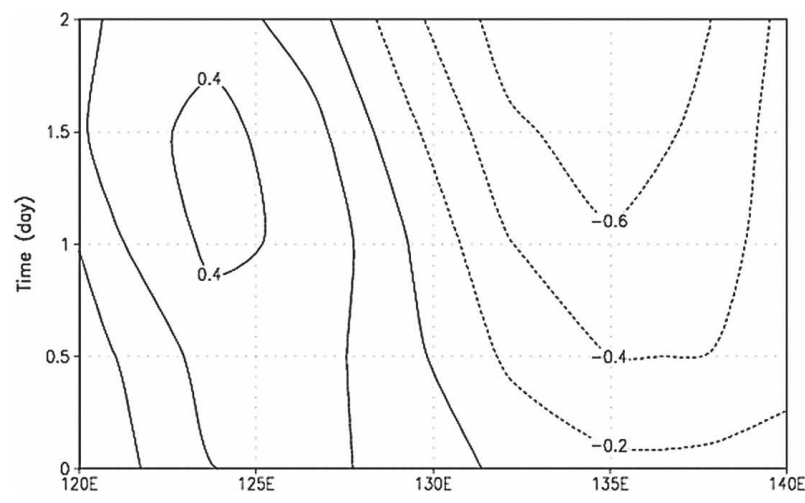

FIG. 7. Time-longitude section of the normalized meridional wind field of the least damped mode at $10^{\circ} \mathrm{N}$ from day 23 to day 25 .

\section{Sensitivity to initial conditions}

To examine the sensitivity of the model solution to initial perturbations, we conducted the following two sensitivity experiments under the realistic summer mean flow and the CFC feedback. In the first experiment the initial perturbation has a much shorter horizontal wavelength but with the same amplitude in vorticity. In the second experiment a westward-propagating equatorial mixed Rossby-gravity wave is specified, based on the analytical solution of Matsuno (1966). The MRG wave is characterized by cross-equatorial flows, and initially its wind amplitude is set to be $0.1 \mathrm{~m} \mathrm{~s}^{-1}$. Figure 8 shows the horizontal patterns of the two initial perturbations.

The numerical results indicate that the final structure of the unstable mode is not sensitive to the initial conditions. In both cases the model reproduces the most unstable modes that have similar synoptic wave train structures in the WNP (Fig. 9). This supports the hypothesis that the summertime synoptic wave train in the WNP is a result of instability of the summer mean flow in the presence of the CFC feedback. Any type of perturbation under such a background condition may grow and develop into the wave train pattern. Note that in reality this background condition (especially the surface moisture condition) may vary on intraseasonal and interannual time scales and, as a result, the synoptic wave train development may be subject to subseasonal and long-term variabilities.

\section{Sensitivity to the vertical shear}

In this section, we further examine the effect of the vertical shear of the mean zonal flow on the growth and propagation characters of the synoptic wave train. To 
(a)

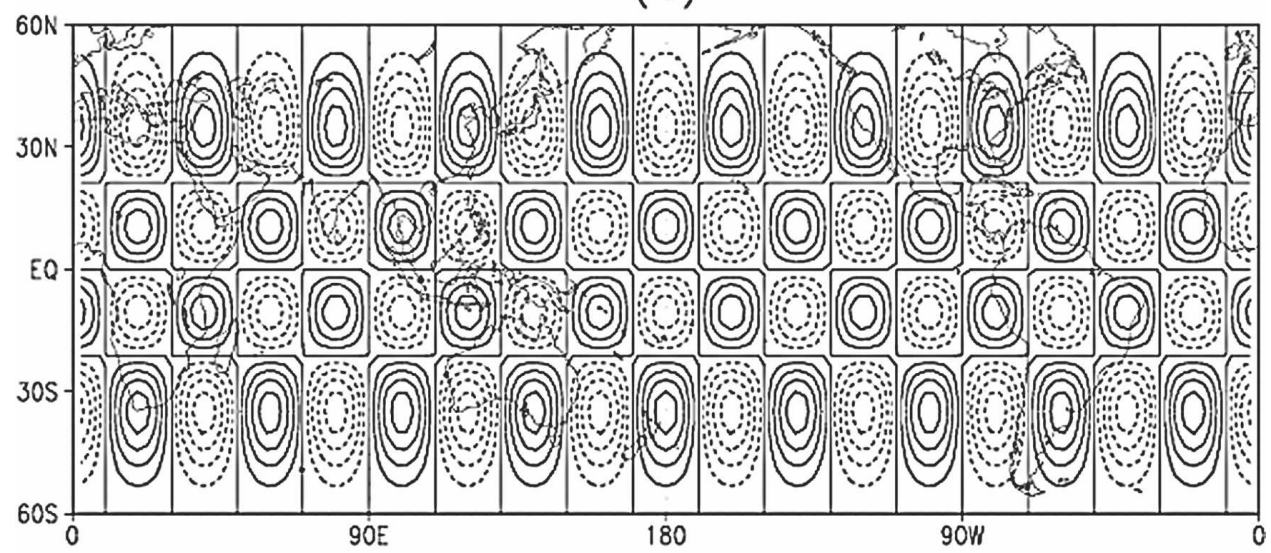

(b)

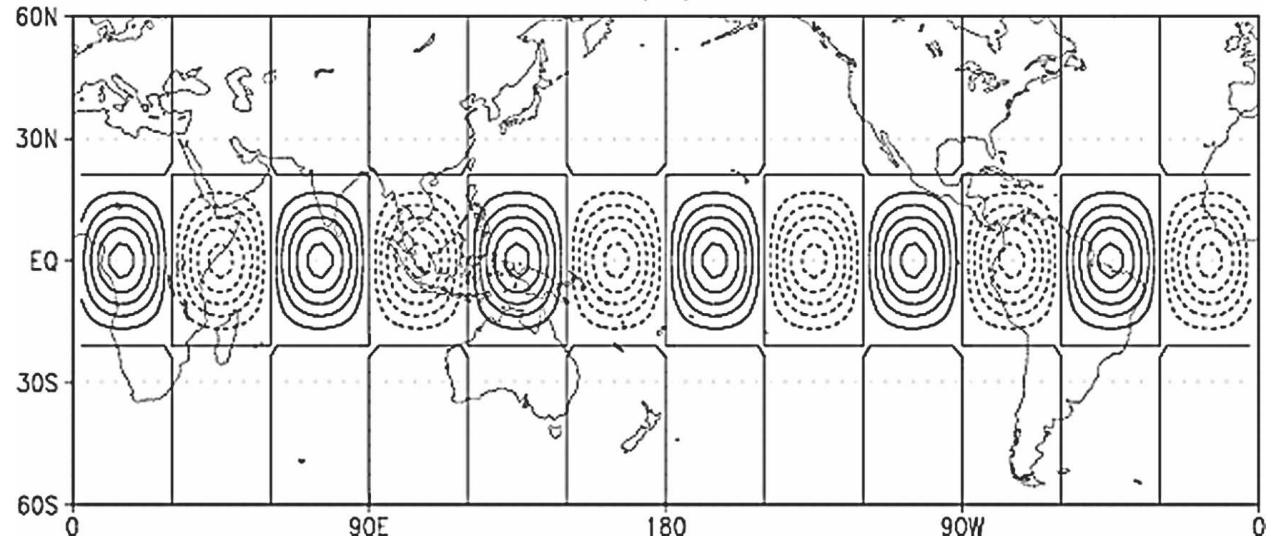

FIG. 8. Two initial perturbation vorticity fields specified in the sensitivity experiments: (a) a zonal wavenumber-9 perturbation and (b) a westward-propagating equatorial mixed Rossby-gravity wave (contour interval is $0.5 \times 10^{-8} \mathrm{~s}^{-1}$ ).

isolate the vertical shear effect, we conducted a set of idealized numerical experiments in which the zonal basic flow is a function of height only and does not change with the zonal and meridional directions. In this idealized setting, both the basic-state meridional wind and vertical motion vanish, and a thermal wind balance holds for the basic-state zonal wind and temperature (or geopotential height) fields. The surface moisture and SST conditions, however, are kept the same as in the control experiment (i.e., the climatological summer mean values) in order to generate the localized growth of perturbation patterns over the WNP.

Three sets of vertical shear experiments were conducted. In the first experiment, an easterly shear of -15 $\mathrm{m} \mathrm{s}^{-1}$ (hereafter the vertical shear is defined as a half of the upper- and lower-tropospheric zonal wind difference) with a linear vertical profile (i.e., $-15,-7.5,0$, $7.5,15 \mathrm{~m} \mathrm{~s}^{-1}$ for $\sigma=0.1,0.3,0.5,0.7,0.9$, respectively) was specified. In the second experiment, a westerly shear of $15 \mathrm{~m} \mathrm{~s}^{-1}$ with the same linear vertical profile was applied. In the third experiment, the vertical shear was set to be zero (i.e., a resting environment). In all three cases the same initial perturbation as in the control experiment (i.e., Fig. 1) was specified.

Figure 10 shows the evolution of maximum perturbation kinetic energy in the three cases. A remarkable asymmetry appears in the perturbation growth. The easterly shear is mostly favorable for the perturbation growth in the WNP, whereas the westerly shear leads to a weaker growth rate. This is consistent with the Rossby wave amplification theory proposed by Wang and Xie (1996), as the low-level circulation is crucial for controlling Ekman pumping and PBL moisture convergence. The zero vertical shear, on the other hand, leads to no growth even though the CFC feedback is considered. This points out the importance of the vertical 
(a)

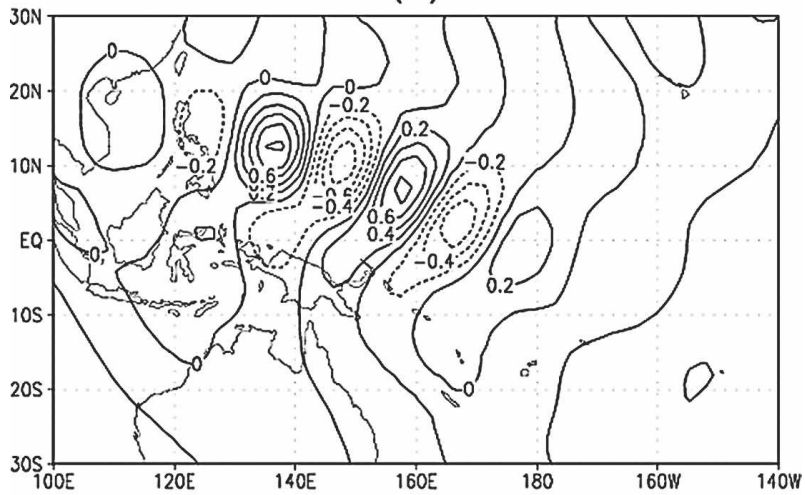

(b)

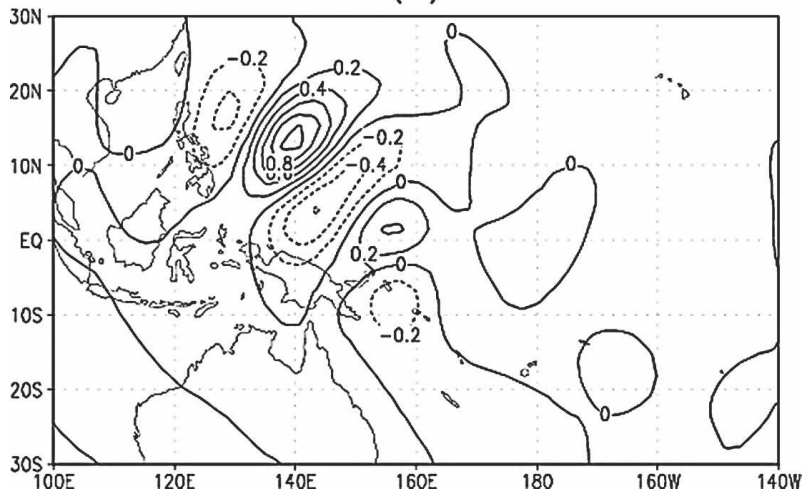

FIG. 9. The normalized meridional wind patterns of the most unstable mode at day 5 calculated from the two initial conditions shown in Fig. 8 in the presence of the 3D summer mean flow and the CFC feedback.

shear in setting up the preferred spatial structure for an efficient convection-circulation feedback.

In addition to the remarkable difference in growth rate, the vertical shear of the mean flow also significantly impacts the wave train propagation character. Figure 11 shows two snapshots (within a 6-h interval) of the geopotential height fields at low level $(\sigma=0.7)$ in the easterly and westerly shear cases. While the easterly shear leads to northwestward propagation of the wave train (Fig. 11a), in contrast the westerly shear leads to southeastward phase propagation (Fig. 11b). In the latter case the perturbation is confined more to the equatorial region.

\section{Conclusions and discussion}

The origin of the summertime synoptic wave train in the western North Pacific is investigated by means of a multilevel baroclinic model in which a realistic 3D summer mean basic state is specified. To mimic the convec-

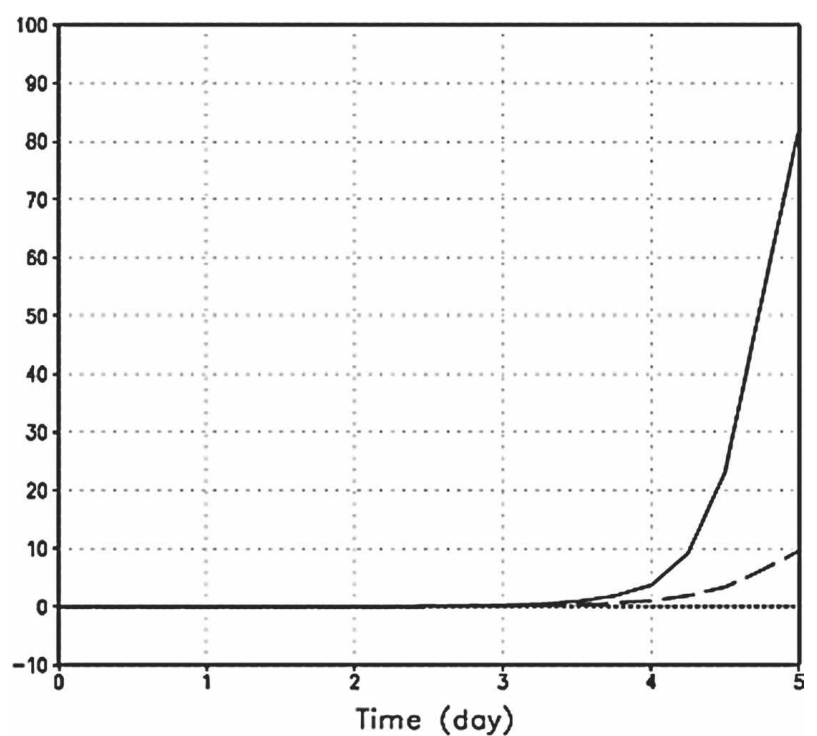

FIG. 10. Evolution of maximum perturbation kinetic energy (unit is $\mathrm{m}^{2} \mathrm{~s}^{-2}$ ) at $\sigma=0.7$ under a constant easterly shear (solid line), westerly shear (dashed line), and zero shear (dotted line).

tion-circulation feedback, a simple convective heating scheme was applied in which the perturbation convective heating is proportional to the PBL moisture convergence. A vertical heating profile with maximum amplitude in the middle (or upper) troposphere is specified, and the heating coefficient depends greatly on the summer mean surface moisture content and SST. By introducing a small perturbation initially, we examine how the perturbation evolves with time under the specified summer mean flow, in the presence or absence of the CFC feedback. The numerical experiments indicate that the origin of the synoptic wave train arises from both the dynamic effect of the 3D summer mean flow and the thermodynamic effect of the convective heating feedback. Lacking either one of the two factors would lead to no growth for the perturbation.

The numerical experiments indicate that the climatological summer mean flow alone is unable to excite baroclinic or barotropic instability to lead to the growth of the tropical perturbations. However, the mean flow determine a preferred length scale for perturbations. In the absence of the CFC feedback, the model reproduces a least damped mode, which has a northwestsoutheast oriented wave train pattern and a typical zonal wavelength of $2500 \mathrm{~km}$. The wave train propagates northwestward at a speed of $2.5^{\circ}$ day $^{-1}$, which is slower than the observed phase speed.

In the presence of both the 3D summer mean flow and the CFC feedback, the model captures a fast growing mode whose horizontal pattern is similar to the observed synoptic wave train in the WNP. The unstable 


\section{(a) Easterly Shear}

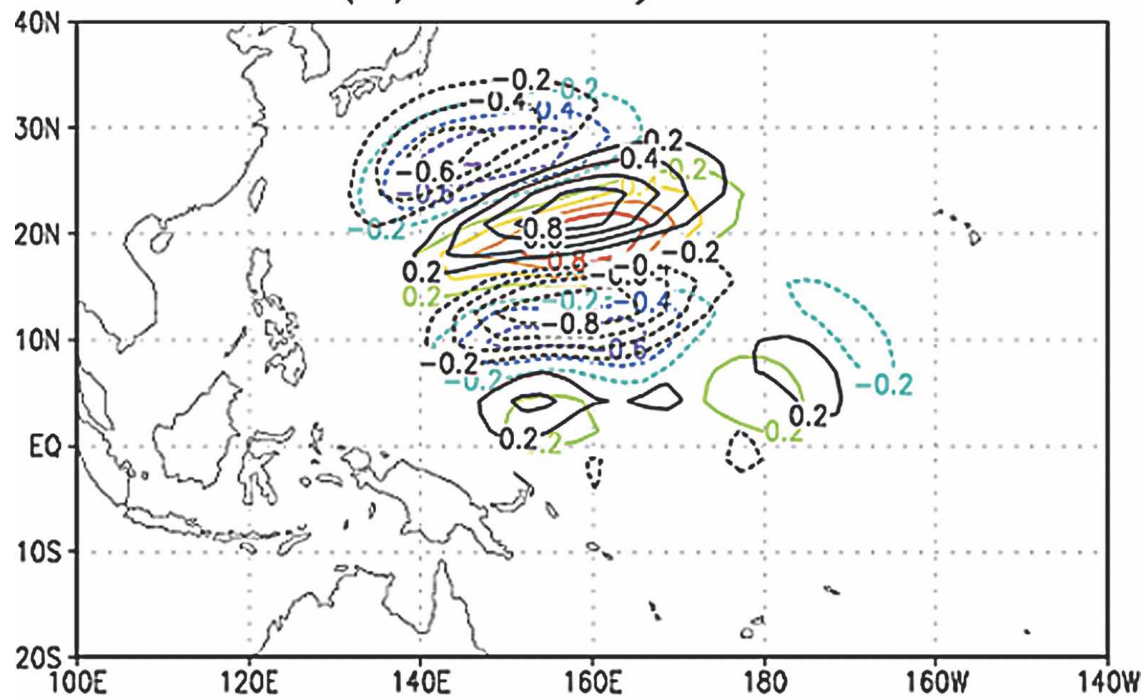

(b) Westerly Shear

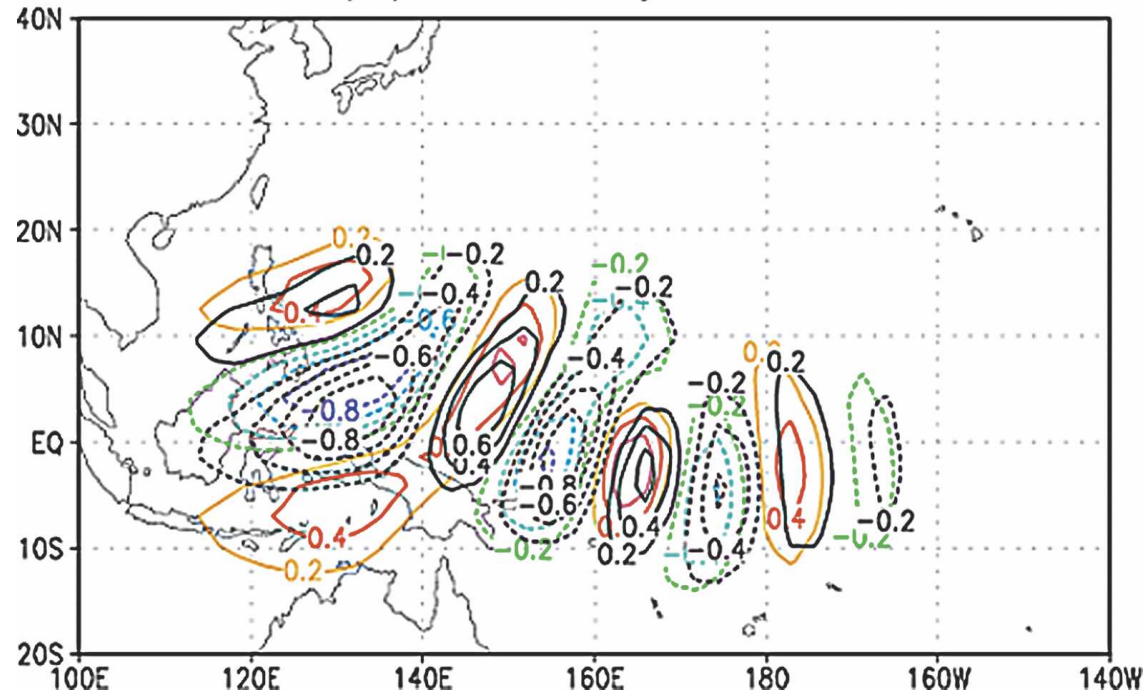

FIG. 11. Two snapshots (at a 6-h interval) of normalized geopotential height fields at $\sigma=0.7$ around day 5 under constant (top) easterly shear and (bottom) westerly shear. In both panels, the height field with color contours appears $6 \mathrm{~h}$ prior to that with black contours.

mode has an eastward tilting vertical profile, propagating northwestward at a phase speed close to the observed.

Why is the western North Pacific the most favorable region for the rapid development of the synoptic wave train in boreal summer? The following two factors may be crucial. First, the warm SST pool and the great water vapor content may lead to a significantly large amount of moist static energy and, thus, conditionally unstable stratification in the region (Li and Wang 1994). Second, the monsoon confluent flow and easterly vertical shear may lead to local energy accumulation (Kuo et al. 2001) and Rossby wave amplification (Wang and Xie 1996) in the lower troposphere. To examine the effect of the two factors, two idealized numerical experiments have been conducted. In the first, 3D summer mean basic flows, along with winter mean SST and surface moisture fields (for the purpose of convective heating calculation only), are specified. In the second, 3D winter mean basic flows along with summer mean SST and surface 
moisture conditions are specified. It turns out that in both cases there is no unstable development of synoptic wave trains in the WNP. The experiments above point out the importance of both the dynamic and thermodynamic factors in determining the preferred region of the rapid development of the synoptic wave disturbances.

The sensitivity experiments with two different initial perturbations, one with a shorter zonal wavelength and the other with an equatorial mixed Rossby-gravity wave structure, indicate that the model solution is not sensitive to the initial conditions. In these simulations, a similar northwest-southeast oriented synoptic wave train develops in the WNP.

Our sensitivity experiments suggest that the vertical shear of the basic flow may have a profound impact on the growth and propagation characters of the synoptic wave train. Given the same surface (moisture and SST) conditions, an easterly shear favors a much faster growth than a westerly shear or zero shear. Whereas the easterly shear leads to northwestward phase propagation, the westerly shear leads to southeastward phase propagation.

In this study with the aid of the numerical experiments we demonstrated the role of the summer mean flow and the CFC feedback in generating the synoptic wave train in the WNP. Keep in mind that in the current model a simple heating scheme is used and the surface moisture is fixed from its climatological seasonal mean value. A further study with more sophisticated convective heating schemes and an interactive moisture field is needed to validate/compare the results presented here. Another issue is how to interpret the dynamic impact of the vertical shear on tropical wave propagation. Currently a theoretical investigation of the role of the basic-state vertical shear in determining tropical wave instability and propagation characters in a 2.5-layer dynamic framework is in progress and results will be reported elsewhere.

Acknowledgments. This work was supported by NSF Grant ATM01-19490 and ONR Grant N000140310739. The International Pacific Research Center is partially sponsored by the Japan Agency for Marine-Earth Science and Technology (JAMSTEC).

\section{APPENDIX}

\section{A Multilevel Baroclinic Model}

The primitive atmospheric governing equations in a vertical sigma coordinate may be written as

$$
\begin{aligned}
& \frac{\partial \mathbf{v}}{\partial t}=-\nabla \phi-R T_{S 0} \nabla P_{S}+\mathbf{G}_{m}, \\
& \frac{\partial T}{\partial t}=\frac{\kappa T_{S 0}}{\sigma} \dot{\sigma}_{D}-\kappa T_{S 0}\langle D\rangle+G_{T}, \\
& \frac{\partial P_{S}}{\partial t}=-\langle D\rangle+G_{C},
\end{aligned}
$$

where $P_{S}=\ln \left(p_{s}\right)$ represents a nature logarithm function of surface pressure, $T_{S 0}=250 \mathrm{~K}$ is a reference atmospheric temperature, $\dot{\sigma}=\dot{\sigma}_{D}+\dot{\sigma}_{A}$ represents vertical velocity in the sigma $\left(\sigma=p / p_{s}\right)$ coordinate, $\dot{\sigma}_{D}=$ $\int_{0}^{\sigma}(D-\langle D\rangle) d \sigma, \dot{\sigma}_{A}=\int_{0}^{\sigma}(\mathbf{v}-\langle\mathbf{v}\rangle) \cdot \nabla P_{S} d \sigma$, and angle brackets denote a vertical average from surface to the top of atmosphere. Here $\mathbf{G}_{m}, G_{T}$, and $G_{C}$ are nonlinear terms representing slow (comparing to gravity wave speed) advective adjustment and atmospheric diabatic processes, which have the following forms:

$$
\begin{aligned}
\mathbf{G}_{m}= & -(\zeta+f) \mathbf{k} \times \mathbf{v}-\dot{\sigma} \frac{\partial \mathbf{v}}{\partial \sigma}-\nabla E-R\left(T-T_{S 0}\right) \nabla P_{S}, \\
G_{T}= & -\mathbf{v} \cdot \boldsymbol{\nabla} T-\dot{\sigma} \frac{\partial T}{\partial \sigma}+Q-\kappa\left(T-T_{S 0}\right)\langle D\rangle \\
& +\frac{\kappa\left(T-T_{S 0}\right)}{\sigma} \dot{\sigma}+\frac{\kappa T_{S 0}}{\sigma} \dot{\sigma}_{A}, \\
G_{C}= & -\langle\mathbf{v}\rangle \cdot \nabla P_{S} .
\end{aligned}
$$

Assume that the basic-state variables satisfy the governing equations above, which implies that the effect of atmospheric transients on the seasonal mean basic state is negligible in the Tropics. By subtracting the basicstate equations from the total equations, one may derive the perturbation governing equations:

$$
\begin{aligned}
& \frac{\partial \mathbf{v}^{\prime}}{\partial t}=-\nabla \phi^{\prime}-R T_{S 0} \nabla P_{S}^{\prime}+\mathbf{G}_{m}^{\prime}, \\
& \frac{\partial T^{\prime}}{\partial t}=\frac{\kappa T_{S 0}}{\sigma} \dot{\sigma}_{D}^{\prime}-\kappa T_{S 0}\left\langle D^{\prime}\right\rangle+G_{T}^{\prime}, \\
& \frac{\partial P_{S}^{\prime}}{\partial t}=-\left\langle D^{\prime}\right\rangle+G_{C}^{\prime},
\end{aligned}
$$

where $\mathbf{G}_{m}^{\prime}=\mathbf{G}_{m}\left(\overline{\mathbf{a}}+\mathbf{a}^{\prime}\right)-\mathbf{G}_{m}(\overline{\mathbf{a}}), \mathbf{G}_{T}^{\prime}=\mathbf{G}_{T}\left(\overline{\mathbf{a}}+\mathbf{a}^{\prime}\right)-$ $\mathbf{G}_{T}(\overline{\mathbf{a}}), \mathbf{G}_{C}^{\prime}=\mathbf{G}_{C}\left(\overline{\mathbf{a}}+\mathbf{a}^{\prime}\right)-\mathbf{G}_{C}(\overline{\mathbf{a}})$, vector a represents model-dependent (both prognostic and diagnostic) variables, and a variable with a prim represents a perturbation field and a variable with an overbar denotes the basic-state field.

To represent the interactive nature of the circulation and convection, a simple convective heating scheme is applied in the model (following Kuo 1974; Wang and Li 1993, 1994) in which the perturbation convective heat- 
ing is primarily determined by the boundary layer moisture convergence, that is,

$$
Q^{\prime}=-\alpha \delta \nabla \cdot\left(\bar{q}_{s} \mathbf{v}_{L}^{\prime}\right) f(\sigma),
$$

where $\bar{q}_{s}$ denotes the summer mean basic-state specific humidity at the surface, $\mathbf{v}_{L}^{\prime}$ represents the lowest-level (PBL) perturbation wind field, $\alpha$ is a heating coefficient representing the strength of the convection-frictional convergence feedback, $\delta$ is a SST-dependent switch-on heating coefficient (Wang and $\mathrm{Li} 1993$ ), and $\delta=1$ when SST $>29.5^{\circ} \mathrm{C}, \delta=0$ when SST $<26.5^{\circ} \mathrm{C}$, and $\delta=(\mathrm{SST}$ - 26.5) $/ 3$ when $26.5^{\circ} \mathrm{C}<\mathrm{SST}<29.5^{\circ} \mathrm{C}$, and $f(\sigma)$ represents the vertical profile of the heating and has a maximum value of a unit in the middle (or upper) troposphere.

For the current study, the heating coefficient, $\alpha$, is set as such that a PBL convergence of $10^{-5} \mathrm{~s}^{-1}$ corresponds to a heating rate of $1^{\circ} \mathrm{C}$ day ${ }^{-1}$. This specified heating rate is in a reasonable range compared to the observed climatological monthly mean value over the ITCZ. Two vertical heating profiles have been tested. In one case the maximum heating is located at $\sigma=0.5$. In the other case the maximum heating resides at $\sigma=0.3$. It turns out that the model solution is not sensitive to the two heating profiles.

\section{REFERENCES}

Aiyyer, A. R., and J. Molinari, 2003: Evolution of mixed Rossbygravity waves in idealized MJO environments. J. Atmos. Sci., 60, 2837-2855.

Chang, C.-P., J.-M. Chen, P. A. Harr, and L. E. Carr, 1996: Northwestward-propagating wave patterns over the tropical western North Pacific during summer. Mon. Wea. Rev., 124, 22452266.

Dickinson, M., and J. Molinari, 2002: Mixed Rossby-gravity waves and western tropical Pacific cyclogenesis. Part I: Synoptic evolution. J. Atmos. Sci., 59, 2183-2196.

Held, I. M., and M. J. Suarez, 1994: A proposal for the intercomparison of the dynamical cores of atmospheric generalcirculation models. Bull. Amer. Meteor. Soc., 75, 1825-1830.

Holland, G. J., 1995: Scale interaction in the western Pacific monsoon. Meteor. Atmos. Phys., 56, 57-79.

Jiang, X., and T. Li, 2005: Reinitiation of the boreal summer intraseasonal oscillation in the tropical Indian Ocean. J. Climate, 18, 377-3795.

- — - and B. Wang, 2004: Structures and mechanisms of the northward propagating boreal summer intraseasonal oscillation. J. Climate, 17, 1022-1039.

Kuo, H.-C., J.-H. Chen, R. T. Williams, and C.-P. Chang, 2001: Rossby wave in zonally opposing mean flow: Behavior in northwest Pacific summer monsoon. J. Atmos. Sci., 58, 1035 1050.

Kuo, H.-L., 1974: Further studies of the parameterization of the influence of cumulus convection on large-scale flow. J. Atmos. Sci., 31, 1231-1240.

Lau, K.-H., and N.-C. Lau, 1990: Observed structure and propagation characteristics of tropical summertime synoptic scale disturbances. Mon. Wea. Rev., 118, 1888-1913.

— , and _ 1992: The energetics and propagation dynamics of tropical summertime synoptic-scale disturbances. Mon. Wea. Rev., 120, 2523-2539.

Li, T., and B. Wang, 1994: The influence of sea surface temperature on the tropical intraseasonal oscillation: A numerical study. Mon. Wea. Rev., 122, 2349-2362.

_ , and B. Fu, 2006: Tropical cyclogenesis associated with Rossby wave energy dispersion of a preexisting typhoon. Part I: Satellite data analyses. J. Atmos. Sci., in press.

,$- \longrightarrow$, X. Ge, B. Wang, and M. Peng, 2003: Satellite data analysis and numerical simulation of tropical cyclone formation. Geophy. Res. Lett., 30, 2122, doi:10.1029/2003GL018556.

— X. Xe, B. Wang, and Y. Zhu, 2006: Tropical cyclogenesis associated with Rossby wave energy dispersion of a preexisting typhoon. Part II: Numerical simulations. J. Atmos. Sci., in press.

Liebmann, B., and H. H. Hendon, 1990: Synoptic-scale disturbances near the equator. J. Atmos. Sci., 47, 1463-1479.

Matsuno, T., 1966: Quasi-geostrophic motions in the equatorial area. J. Meteor. Soc. Japan, 44, 25-43.

Nitta, T., and M. Yanai, 1969: A note on the barotropic instability of the tropical easterly current. J. Meteor. Soc. Japan, 47, 127-130.

Tai, K. S., and Y. Ogura, 1987: An observational study of easterly waves over the eastern Pacific in the northern summer using FGGE data. J. Atmos. Sci., 44, 339-361.

Takayabu, Y. N., and T. Nitta, 1993: 3-5 day disturbances coupled with convection in the tropical Pacific Ocean. J. Meteor. Soc. Japan, 71, 221-245.

Tam, C.-Y., and T. Li, 2006: The origin and dispersion characteristics of the observed tropical summertime synoptic-scale waves over the western Pacific. Mon. Wea. Rev., in press.

Ting, M., and L. Yu, 1998: Steady response to tropical heating in wavy linear and nonlinear baroclinic models. J. Atmos. Sci., $\mathbf{5 5}$, 3565-3582.

Wang, B., and T. Li, 1993: A simple tropical atmospheric model of relevance to short-term climate variations. J. Atmos. Sci., 50, $260-284$

_ and - 1994: Convective interaction with boundary-layer dynamics in the development of a tropical intraseasonal system. J. Atmos. Sci., 51, 1386-1400.

—_, and X. Xie, 1996: Low-frequency equatorial waves in sheared zonal flow. Part I: Stable waves. J. Atmos. Sci., 53, 449-467.

—, R. Wu, and T. Li, 2003: Atmosphere-warm ocean interaction and its impact on Asian-Australian monsoon variation. J. Climate, 16, 1195-1211. 